\title{
Superscaling and charge-changing neutrino scattering from nuclei in the $\Delta$ region beyond the relativistic Fermi gas model
}

\author{
M. V. Ivanov, ${ }^{1}$ M. B. Barbaro, ${ }^{2}$ J. A. Caballero, ${ }^{3}$ A. N. Antonov, ${ }^{1}$ E. Moya de Guerra,,${ }^{4,5}$ and M. K. Gaidarov ${ }^{1,5}$ \\ ${ }^{1}$ Institute for Nuclear Research and Nuclear Energy, Bulgarian Academy of Sciences, Sofia 1784, Bulgaria \\ ${ }^{2}$ Dipartimento di Fisica Teorica, Università di Torino and INFN, Sezione di Torino, Via P. Giuria 1, I-10125 Torino, Italy \\ ${ }^{3}$ Departamento de Física Atómica, Molecular y Nuclear, Universidad de Sevilla, Apdo. 1065, 41080 Sevilla, Spain \\ ${ }^{4}$ Departamento de Fisica Atómica, Molecular y Nuclear, Facultad de Ciencias Fisicas, Universidad Complutense de Madrid, \\ E-28040 Madrid, Spain \\ ${ }^{5}$ Instituto de Estructura de la Materia, CSIC, Serrano 123, E-28006 Madrid, Spain
}

(Received 5 October 2007; published 28 March 2008)

\begin{abstract}
The superscaling analysis using the scaling function obtained within the coherent density fluctuation model is extended to calculate charge-changing neutrino and antineutrino scattering on ${ }^{12} \mathrm{C}$ at energies from 1 to $2 \mathrm{GeV}$ not only in the quasielastic but also in the $\Delta$ excitation region. The results are compared with those obtained using the scaling functions from the relativistic Fermi gas model and from the superscaling analysis of inclusive scattering of electrons from nuclei.
\end{abstract}

DOI: 10.1103/PhysRevC.77.034612

PACS number(s): 25.30.Pt, 23.40.Bw, 24.10.-i, 21.60.-n

\section{INTRODUCTION}

The analyses of scaling (e.g., Refs. [1-10]) and superscaling (e.g., [10-21]) phenomena observed in electron scattering from nuclei and the consideration on the same basis of neutrino (antineutrino)-nucleus scattering have been among the important tasks of nuclear physics in the past few decades. Scalings of the first and the second kind (a very weak dependence of the reduced cross section on the momentum transfer $q$ and on the mass number, respectively) at excitation energies below the quasielastic (QE) peak turn out to be related to the high-momentum components of the nucleon momentum distribution $n(k)$ at $k>2 \mathrm{fm}^{-1}$ that are similar for all nuclei and are due to the short-range and tensor correlations in the nuclei. One says that the reduced cross sections exhibit superscaling when both types of scaling occur. The violation of the scaling of the first kind above the QE peak is related to the excitation of a nucleon in the nucleus to a $\Delta$ resonance [15,22] and to effects of the meson exchange currents [23-27]. The first theoretical explanations of the superscaling have been given in Refs. $[10,11]$ in the framework of the relativistic Fermi gas (RFG) model. The analyses of the world data on inclusive electron-nucleus scattering in Refs. [12,13] confirmed the observation of this phenomenon, but simultaneously they showed the necessity to consider it on the basis of more complex dynamical picture of finite nuclear systems beyond the RFG. The main reason for this is that the scaling function in the RFG model is $f_{\mathrm{RFG}}^{\mathrm{QE}}\left(\psi^{\prime}\right)=0$ for $\psi^{\prime} \leqslant-1$, whereas the experimental scaling function extracted from $\left(e, e^{\prime}\right)$ data extends to large negative values of the scaling variable $\psi^{\prime}$ up to $\psi^{\prime} \approx-2$ where effects beyond the mean-field approximation are important. A theoretical approach that correctly interprets superscaling in the $\psi^{\prime} \leqslant 0$ region is the coherent density fluctuation model (CDFM) (e.g., Refs. [28,29]). This model represents a natural extension of the Fermi gas model to realistic nuclear systems and it is based on the generator coordinate method [30]. In the CDFM the QE scaling function $f\left(\psi^{\prime}\right)$ is related to realistic nucleon momentum and density distributions and it agrees with the data for negative values of $\psi^{\prime}$, including $\psi^{\prime} \lesssim-1[16-20]$. This is so because the CDFM momentum distribution is not a sharp function of $k$ as the RFG one is. Reliable separation of inclusive electron scattering data into their longitudinal and transverse contributions for $A>4$ nuclei made it possible to obtain (see, e.g., Refs. [12,13,15,31]) from the data a "universal" phenomenological QE scaling function $f^{\mathrm{QE}}\left(\psi^{\prime}\right)$. In the present work we use the fit of Ref. [14], which is based on the experimental analysis of J. Jourdan [32]. A striking feature of the scaling function $f^{\mathrm{QE}}\left(\psi^{\prime}\right)$ extracted from the superscaling analysis (SuSA) is its asymmetric shape with respect to the peak position $\psi^{\prime}=0$ with a pronounced tail extended toward positive $\psi^{\prime}$ values. This is in contrast to the scaling function in the RFG model that is symmetric with respect to $\psi^{\prime}=0$. This property of the phenomenological SuSA scaling function imposed further theoretical considerations. A detailed investigation of such asymmetry has been presented in Refs. [33-35] in the context of the relativistic mean-field (RMF) approach. These studies have proved the crucial role played by the description of final-state interactions (FSI), through the RMF, to reproduce adequately the asymmetric shape shown by the data analysis.

The approach of SuSA to the QE electron scattering (at energies from several hundred $\mathrm{MeV}$ to a few $\mathrm{GeV}$ ) have been extended to include also processes in which $\Delta$ excitation dominates [31]. In the CDFM this was done in Ref. [19].

The validity of superscaling in inclusive electron scattering allowed one to start studies of neutrino (antineutrino) scattering off nuclei on the same basis ( $[31,33,36,37])$. Given the corresponding scaling functions, the cross sections of charge-changing (CC) [33] or neutral-current (NC) [27] neutrino (antineutrino), nucleus-scattering cross sections for intermediate to high energies can be obtained by multiplying the elementary single-nucleon (s.n.) $\mathrm{CC}$ or $\mathrm{NC}$ neutrino (antineutrino) cross sections by the corresponding scaling function. This procedure relies on some assumptions that have recently been tested within the RMF+FSI model and are related to the isospin degrees of freedom [38]. 
A number of other theoretical studies of CC (e.g., Refs. [3948]) and NC (e.g., [39,40,47,49-52]) neutrino (antineutrino)nucleus scattering has also been developed in recent years.

In the QE region the CDFM scaling function (with asymmetry introduced in [19]) has been applied to analyze charge-changing neutrino (antineutrino) scattering on ${ }^{12} \mathrm{C}$ (for energies of the incident particles from 1 to $2 \mathrm{GeV}$ ) in Ref. [19] and neutral current neutrino (antineutrino) scattering on the same nucleus with proton and neutron knockout in Ref. [53]. The results were compared with those from the RFG model and from the superscaling analysis (SuSA) [15,31]. These analyses made it possible to gain information simultaneously and on the same footing about the role of both the local density and the momentum distribution in nuclei for the description of superscaling and of electron- and neutrino-nucleus scattering (e.g., Refs. [17,19]). One of the advantages of the superscaling analysis within the CDFM was to find the relationship [17] between the behavior of the scaling function for negative values of $\psi^{\prime}$ and the slope of the nucleon momentum distribution $n(k)$ at higher values of the momentum $(k>$ $1.5 \mathrm{fm}^{-1}$ ) that is similar for all nuclei due to the short-range nucleon-nucleon correlations. It became possible to show the sensitivity of the calculated CDFM scaling function to the peculiarities of $n(k)$ in different regions of the momentum [17]. It was also shown that the existing data on the $\psi^{\prime}$ scaling are informative for $n(k)$ at momenta up to $k \leqslant 2-2.5 \mathrm{fm}^{-1}$.

The aim of this work is to extend the CDFM scaling approach from the QE region to the $\Delta$ region for $\mathrm{CC}$ neutrino and antineutrino scattering from nuclei using a constructed realistic CDFM scaling function for the same region.

The article is organized in the following way: the theoretical scheme is given in Sec. II. It includes the main relationships of the CDFM scaling functions in both the $\mathrm{QE}$ and $\Delta$ regions as well as a brief outline of the formalism for CC neutrino scattering. The results for ${ }^{12} \mathrm{C}\left(v_{\mu}, \mu^{-}\right)$and ${ }^{12} \mathrm{C}\left(\bar{v}_{\mu}, \mu^{+}\right)$reaction cross sections are presented and discussed in Sec. III. The conclusions are summarized in Sec. IV.

\section{THE THEORETICAL SCHEME}

\section{A. CDFM scaling function in the $\mathrm{QE}$ region}

The QE CDFM scaling function was obtained [16-19] on the basis of the local density distribution, $\rho(r)$, as well as on the basis of the nucleon momentum distribution, $n(k)$. It is expressed by the sum of the proton $f_{p}^{\mathrm{QE}}\left(\psi^{\prime}\right)$ and neutron $f_{n}^{\mathrm{QE}}\left(\psi^{\prime}\right)$ scaling functions, which are determined by the proton and neutron densities, $\rho_{p}(r)$ and $\rho_{n}(r)$ [or by corresponding momentum distributions $n_{p}(k)$ and $n_{n}(k)$ ], respectively [19]:

$$
f^{\mathrm{QE}}\left(\psi^{\prime}\right)=\frac{1}{A}\left[Z f_{p}^{\mathrm{QE}}\left(\psi^{\prime}\right)+N f_{n}^{\mathrm{QE}}\left(\psi^{\prime}\right)\right] .
$$

The proton and neutron scaling functions in Eq. (1) are presented as sums of scaling functions for negative $\left[f_{p(n), 1}^{\mathrm{QE}}\left(\psi^{\prime}\right)\right]$ and positive $\left[f_{p(n), 2}^{\mathrm{QE}}\left(\psi^{\prime}\right)\right]$ values of $\psi^{\prime}$ :

$$
f_{p(n)}^{\mathrm{QE}}\left(\psi^{\prime}\right)=f_{p(n), 1}^{\mathrm{QE}}\left(\psi^{\prime}\right)+f_{p(n), 2}^{\mathrm{QE}}\left(\psi^{\prime}\right),
$$

where (in the case when the scaling function is obtained on the basis of the density distributions)

$$
\begin{aligned}
f_{p(n), 1}^{\mathrm{QE}}\left(\psi^{\prime}\right) & =\int_{0}^{\alpha_{p(n)} /\left(k_{F}^{p(n)}\left|\psi^{\prime}\right|\right)} d R\left|F_{p(n)}(R)\right|^{2} f_{\mathrm{RFG}, 1}^{p(n)}\left[\psi^{\prime}(R)\right], \\
\psi^{\prime} & \leqslant 0, \\
f_{p(n), 2}^{\mathrm{QE}}\left(\psi^{\prime}\right) & =\int_{0}^{c_{2} \alpha_{p(n)} /\left(k_{F}^{p(n)} \psi^{\prime}\right)} d R\left|F_{p(n)}(R)\right|^{2} f_{\mathrm{RFG}, 2}^{p(n)}\left[\psi^{\prime}(R)\right], \\
\psi^{\prime} & \geqslant 0
\end{aligned}
$$

with

$$
f_{\mathrm{RFG}, 1}^{p(n)}\left[\psi^{\prime}(R)\right]=c_{1}\left[1-\left(\frac{k_{F}^{p(n)} R\left|\psi^{\prime}\right|}{\alpha_{p(n)}}\right)^{2}\right], \quad \psi^{\prime} \leqslant 0
$$

and with two forms of $f_{\mathrm{RFG}, 2}^{p(n)}\left[\psi^{\prime}(R)\right]$ : a parabolic form,

$$
f_{\mathrm{RFG}, 2}^{p(n)}\left[\psi^{\prime}(R)\right]=c_{1}\left[1-\left(\frac{k_{F}^{p(n)} R \psi^{\prime}}{c_{2} \alpha_{p(n)}}\right)^{2}\right], \quad \psi^{\prime} \geqslant 0
$$

and an exponential form,

$$
f_{\mathrm{RFG}, 2}^{p(n)}\left[\psi^{\prime}(R)\right]=c_{1} \exp \left[-\frac{k_{F}^{p(n)} R \psi^{\prime}}{c_{2} \alpha_{p(n)}}\right], \quad \psi^{\prime} \geqslant 0 .
$$

The normalizations of the functions are:

$$
\begin{gathered}
\int_{0}^{\infty}\left|F_{p(n)}(R)\right|^{2} d R=1, \\
\int_{-\infty}^{\infty} f_{p(n)}^{\mathrm{QE}}\left(\psi^{\prime}\right) d \psi^{\prime}=1, \\
\int_{-\infty}^{\infty} f^{\mathrm{QE}}\left(\psi^{\prime}\right) d \psi^{\prime}=1 .
\end{gathered}
$$

It can be seen that due to the normalization conditions (9) and (10) the two parameters $c_{1}$ and $c_{2}$ are not independent. In the case of the parabolic form of $f_{\mathrm{RFG}, 2}^{p(n)}$ [Eq. (6)] $c_{2}=\frac{3}{2 c_{1}}-1$ and in the case of the exponential form [Eq. (7)] $c_{2}=\frac{1-(2 / 3) c_{1}}{0.632 c_{1}}$.

In Eqs. (3) and (4) the proton and neutron weight functions are obtained from the proton and neutron densities, respectively:

$$
\begin{aligned}
\left|F_{p(n)}(R)\right|^{2} & =-\left.\frac{4 \pi R^{3}}{3 Z(N)} \frac{d \rho_{p(n)}(r)}{d r}\right|_{r=R}, \\
\alpha_{p(n)} & =\left[\frac{9 \pi Z(N)}{4}\right]^{1 / 3},
\end{aligned}
$$

with normalization

$$
\int_{0}^{\infty} \rho_{p(n)}(\mathbf{r}) d \mathbf{r}=Z(N) .
$$

In the CDFM the Fermi momentum for the protons and neutrons can be calculated using the expression

$$
k_{F}^{p(n)}=\alpha_{p(n)} \int_{0}^{\infty} d R \frac{1}{R}\left|F_{p(n)}(R)\right|^{2} .
$$

The QE electron scattering was considered within the CDFM in Ref. [19]. Two types of experimental data were considered. In the first one the transferred momentum in the position of the maximum of the QE peak extracted from data $\left(\omega_{\mathrm{exp}}^{\mathrm{QE}}\right)$ is $q_{\mathrm{exp}}^{\mathrm{QE}} \geqslant 450 \mathrm{MeV} / c \approx 2 k_{F}$ and thus corresponds to the domain where scaling is fulfilled [15,31]. It was found 
by fitting to the maximum of the QE peak extracted from data the value of $c_{1}$ to be $0.72-0.73$, i.e., that it is similar to that in the RFG model case (case of symmetry of the RFG and of the CDFM QE scaling functions with $c_{1}=0.75$ ). This leads to an almost symmetric form of the CDFM scaling function for cases in which $q_{\exp }^{\mathrm{QE}} \geqslant 450 \mathrm{MeV} / c$. In the second type of experimental data $q_{\exp }^{\mathrm{QE}}$ is not in the scaling region $\left(q_{\mathrm{exp}}^{\mathrm{QE}} \leqslant 450 \mathrm{MeV} / c \approx 2 k_{F}\right)$. For them it was found by fitting to the maximum of the QE peak the value of $c_{1}$ to be 0.63 . For these cases the scaling function in the CDFM is definitely asymmetric. It was shown in Ref. [19] that the results for the almost-symmetric CDFM scaling function $f^{\mathrm{QE}}\left(\psi^{\prime}\right)$ with $c_{1}=0.72$ agree with the data in the region of the QE peak in cases when $q_{\exp }^{\mathrm{QE}} \geqslant 450 \mathrm{MeV} / c \approx 2 k_{F}$ and overestimates them when $q_{\exp }^{\mathrm{QE}} \leqslant 450 \mathrm{MeV} / c$. The results obtained when an asymmetric scaling function $f^{\mathrm{QE}}\left(\psi^{\prime}\right)$ with $f_{\mathrm{RFG}, 2}^{p(n)}\left[\psi^{\prime}(R)\right]$ from Eq. (6) and the value $c_{1}=0.63$ are used agree with the data in cases when $q_{\mathrm{exp}}^{\mathrm{QE}} \leqslant 450 \mathrm{MeV} / c \approx 2 k_{F}$ and underestimate them when $q_{\exp }^{\mathrm{QE}} \geqslant 450 \mathrm{MeV} / c$ in the region close to the QE peak. So, we pointed out that the two different values of $c_{1}(0.72$ and 0.63 ) found by the fitting to the position of $q_{\exp }^{\mathrm{QE}}$ [and the corresponding to them almost symmetric and definitely asymmetric forms of the CDFM scaling function $\left.f^{\mathrm{QE}}\left(\psi^{\prime}\right)\right]$ are in relation to that whether $q_{\exp }^{\mathrm{QE}}$ is in the domain of the scaling $\left(q_{\exp }^{\mathrm{QE}} \geqslant 2 k_{F}\right)$ or it is not $\left(q_{\exp }^{\mathrm{QE}} \leqslant 2 k_{F}\right)$. In connection to this consideration, in Ref. [19] we showed that the cross section results for CC neutrino (antineutrino) scattering on ${ }^{12} \mathrm{C}$ using the asymmetric QE CDFM scaling function $f^{\mathrm{QE}}\left(\psi^{\prime}\right)$ $\left(c_{1}=0.63\right)$ for incident energies from 1 to $2 \mathrm{GeV}$ are close to those of SuSA [15,31] and are different from the RFG model (where $c_{1}=0.75$ ) results.

\section{B. CDFM scaling function in the $\Delta$ region}

The CDFM scaling analysis was extended in Ref. [19] to the $\Delta$-peak region. The CDFM scaling function was written in the form:

$$
f^{\Delta}\left(\psi_{\Delta}^{\prime}\right)=\int_{0}^{\infty} d R\left|F_{\Delta}(R)\right|^{2} f_{\mathrm{RFG}}^{\Delta}\left[\psi_{\Delta}^{\prime}(R)\right],
$$

where the RFG scaling function in the $\Delta$ domain is given by Ref. [31]:

$$
f_{\mathrm{RFG}}^{\Delta}\left(\psi_{\Delta}^{\prime}\right)=\frac{3}{4}\left(1-\psi_{\Delta}^{\prime 2}\right) \theta\left(1-\psi_{\Delta}^{\prime 2}\right)
$$

and the weight function $\left|F_{\Delta}(R)\right|^{2}$ is related to the density distribution:

$$
\left|F_{\Delta}(R)\right|^{2}=-\left.\frac{4 \pi R^{3}}{3 A} \frac{d \rho(r)}{d r}\right|_{r=R} .
$$

In Eqs. (15) and (16) the shifted scaling variable $\psi_{\Delta}^{\prime}$ is expressed by (see, e.g., Ref. [31]):

$\psi_{\Delta}^{\prime} \equiv\left[\frac{1}{\xi_{F}}\left(\kappa \sqrt{\rho_{\Delta}^{\prime 2}+\frac{1}{\tau^{\prime}}}-\lambda^{\prime} \rho_{\Delta}^{\prime}-1\right)\right]^{1 / 2}\left\{\begin{array}{l}+1, \lambda^{\prime} \geqslant \lambda^{\prime 0} \\ -1, \lambda^{\prime} \leqslant \lambda_{\Delta}^{\prime 0}\end{array}\right.$, where

$$
\begin{aligned}
\xi_{F} & \equiv \sqrt{1+\eta_{F}^{2}}-1, \quad \eta_{F} \equiv \frac{k_{F}}{m_{N}} \\
\lambda^{\prime} & =\lambda-\frac{E_{\text {shift }} 2 m_{N}}{2} \quad \tau^{\prime}=\kappa^{2}-\lambda^{\prime 2}, \\
\lambda & =\frac{\omega}{2 m_{N}}, \quad \kappa=\frac{q}{2 m_{N}}, \quad \tau=\kappa^{2}-\lambda^{2}, \\
\lambda_{\Delta}^{\prime 0} & =\lambda_{\Delta}^{0}-\frac{E_{\text {shift }}}{2 m_{N}}, \quad \lambda_{\Delta}^{0}=\frac{1}{2}\left[\sqrt{\mu_{\Delta}^{2}+4 \kappa^{2}}-1\right], \\
\mu_{\Delta} & =m_{\Delta} / m_{N}, \\
\rho_{\Delta} & =1+\frac{\left(\mu_{\Delta}^{2}-1\right)}{4 \tau}, \quad \rho_{\Delta}^{\prime}=1+\frac{\left(\mu_{\Delta}^{2}-1\right)}{4 \tau^{\prime}} .
\end{aligned}
$$

$q$ and $\omega$ being the transferred momentum and energy, and $m_{\Delta}$ and $m_{N}$ the masses of the $\Delta$ resonance and the nucleon, respectively.

In Eq (15):

$$
\psi_{\Delta}^{\prime 2}(R)=\frac{\kappa \sqrt{\rho_{\Delta}^{\prime 2}+\frac{1}{\tau^{\prime}}}-\lambda^{\prime} \rho_{\Delta}^{\prime}-1}{\sqrt{1+\frac{k_{F}^{2}(R)}{m_{N}^{2}}}-1} \equiv t(R) \psi_{\Delta}^{\prime 2},
$$

where

$$
t(R) \equiv \frac{\sqrt{1+\frac{k_{F}^{2}}{m_{N}^{2}}}-1}{\sqrt{1+\frac{k_{F}^{2}(R)}{m_{N}^{2}}}-1}, \quad k_{F}(R)=\frac{\alpha}{R}
$$

and

$$
\alpha=\left(\frac{9 \pi A}{8}\right)^{1 / 3} .
$$

In the CDFM $k_{F}$ can be calculated using the density distribution:

$$
k_{F}=\alpha \int_{0}^{\infty} d R \frac{1}{R}\left|F_{\Delta}(R)\right|^{2},
$$

where $\left|F_{\Delta}(R)\right|$ is given by Eq. (17) and $\alpha$ by Eq. (27). In an equivalent formulation of the CDFM, proposed in Ref. [17], the scaling function and the Fermi momentum can be obtained using the nucleon momentum distribution.

It was shown in Ref. [19] that though the functional forms of $f^{\Delta}\left(\psi_{\Delta}^{\prime}\right)$ [Eq. (15)], the weight function $\left|F_{\Delta}(R)\right|^{2}$ [Eq. (17)] and of $k_{F}$ [Eq. (28)] are like in the QE region (see Eqs. (3), (4), (11), and (14), respectively), it cannot be expected that the parameters of the densities when a $\Delta$ resonance is excited (e.g. the half-radius $R_{\Delta}$ and the diffuseness $b_{\Delta}$ when Fermi-type distributions have been used) will be equal to the values of $R$ and $b$ in the QE case. Indeed the scaling data of the $\Delta$ peak extracted from the high-quality world data for inclusive electron scattering (given in Ref. [31]) can be fitted by using for ${ }^{12} \mathrm{C}$ the effective values $R_{\Delta}=1.565 \mathrm{fm}$ and $b_{\Delta}=$ $0.420 \mathrm{fm}$ and a coefficient in the right-hand side of Eq. (16) for the RFG scaling function $f_{\mathrm{RFG}}^{\Delta}\left(\psi_{\Delta}^{\prime}\right)$ equal to 0.54 instead of 3/4. The value of the Fermi momentum $k_{F}=1.20 \mathrm{fm}^{-1}$ ensures the normalization to unity of the function $f_{\mathrm{RFG}}^{\Delta}\left(\psi_{\Delta}^{\prime}\right)$. As can be seen, the value of $R_{\Delta}$ is smaller than that in the description of the QE superscaling function for ${ }^{12} \mathrm{C}[16,17]$ 


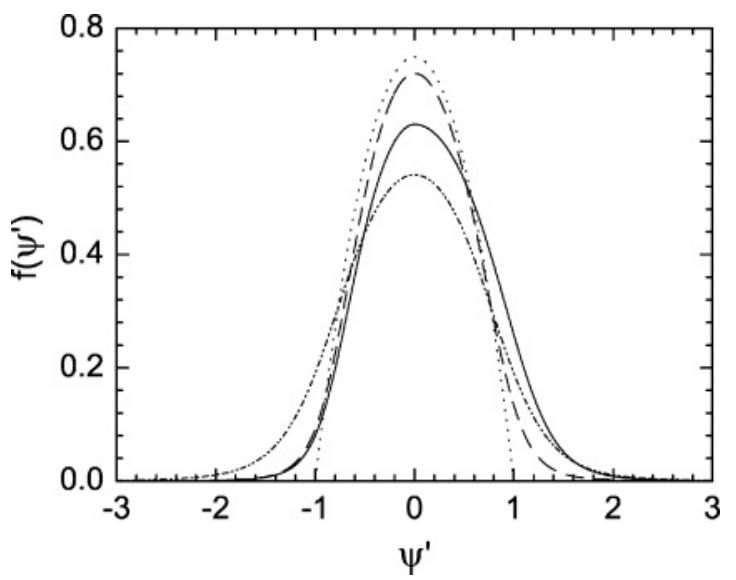

FIG. 1. The CDFM scaling function in the $\Delta$ region $f^{\Delta}\left(\psi_{\Delta}^{\prime}\right)$ (double-dot dashed line) calculated with $R_{\Delta}=1.565 \mathrm{fm}, b_{\Delta}=$ $0.420 \mathrm{fm}, k_{F}=1.20 \mathrm{fm}^{-1}$, and a coefficient in the right-hand side of Eq. (16) equal to 0.54 (instead of 3/4). By dotted, dashed, and solid lines are presented the QE-scaling functions $f^{\mathrm{QE}}\left(\psi_{\mathrm{OE}}^{\prime}\right)$ in the RFG model and in the CDFM with $c_{1}=0.72$ and $c_{1}=0.63$, respectively.

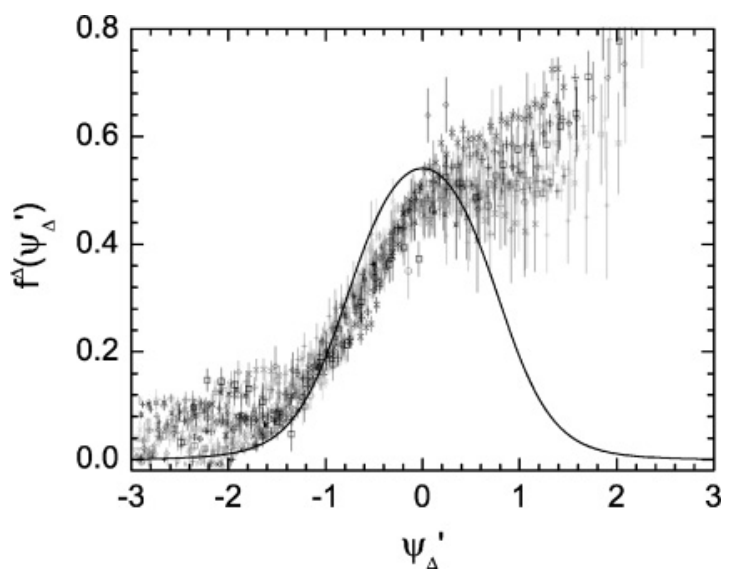

FIG. 2. The same as in Fig. 1 for the CDFM scaling function $f^{\Delta}\left(\psi_{\Delta}^{\prime}\right)$ in the $\Delta$ region (solid line). Averaged experimental values of $f^{\Delta}\left(\psi_{\Delta}^{\prime}\right)$ are taken from Ref. [31].

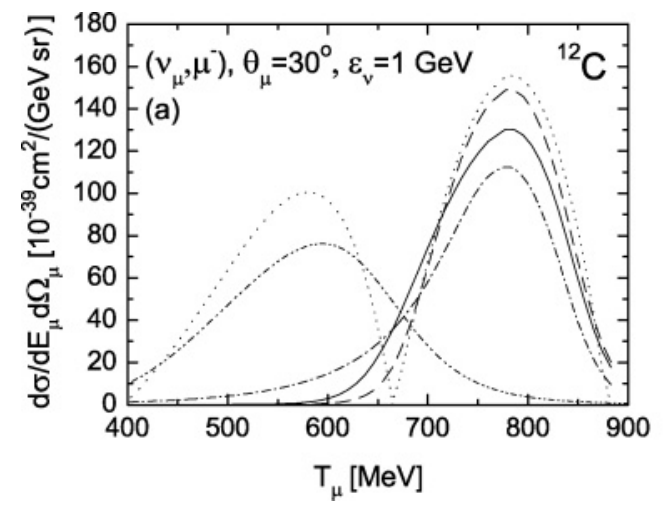

( $R=2.470 \mathrm{fm}$ ), whereas the value of $b_{\Delta}$ is the same as $b$ in the QE case.

\section{Scaling functions and charge-changing neutrino-nucleus reaction cross section}

Here we present applications of the CDFM QE- and $\Delta$-scaling function to the calculations of $\mathrm{CC}$ neutrino-nucleus reaction cross sections. We follow the formalism given in Ref. [31]. The CC neutrino cross section in the target laboratory frame is given in the form

$$
\left(\frac{d^{2} \sigma}{d \Omega d k^{\prime}}\right)_{\chi} \equiv \sigma_{0} \mathcal{F}_{\chi}^{2},
$$

where $\chi=+$ for neutrino-induced reactions (for example, $v_{l}+n \rightarrow \ell^{-}+p$, where $\left.\ell=e, \mu, \tau\right)$ and $\chi=-$ for antineutrino-induced reactions (for example, $\overline{\nu_{l}}+p \rightarrow \ell^{+}+$ $n)$,

$$
\sigma_{0} \equiv \frac{\left(G \cos \theta_{c}\right)^{2}}{2 \pi^{2}}\left(k^{\prime} \cos \tilde{\theta} / 2\right)^{2},
$$

where $G=1.16639 \times 10^{-5} \mathrm{GeV}^{-2}$ is the Fermi constant and $\theta_{c}$ is the Cabibbo angle $\left(\cos \theta_{c}=0.9741\right)$,

$$
\begin{aligned}
\tan ^{2} \tilde{\theta} / 2 & \equiv \frac{\left|Q^{2}\right|}{v_{0}}, \\
v_{0} & \equiv\left(\epsilon+\epsilon^{\prime}\right)^{2}-q^{2}=4 \epsilon \epsilon^{\prime}-\left|Q^{2}\right| .
\end{aligned}
$$

The function $\mathcal{F}_{\chi}^{2}$ depends on the nuclear structure and can be written as [31]:

$$
\begin{aligned}
\mathcal{F}_{\chi}^{2}= & {\left[\widehat{V}_{\mathrm{CC}} R_{\mathrm{CC}}+2 \widehat{V}_{\mathrm{CL}} R_{\mathrm{CL}}+\widehat{V}_{\mathrm{LL}} R_{\mathrm{LL}}+\widehat{V}_{\mathrm{T}} R_{\mathrm{T}}\right] } \\
& +\chi\left[2 \widehat{V}_{\mathrm{T}^{\prime}} R_{\mathrm{T}^{\prime}}\right]
\end{aligned}
$$

that is, as a generalized Rosenbluth decomposition having charge-charge (CC), charge-longitudinal (CL), longitudinallongitudinal (LL), and two types of transverse $\left(T, T^{\prime}\right)$ responses $(R$ 's) with the corresponding leptonic kinematical factors ( $V$ 's) presented in Ref. [31]. The nuclear response functions in both $\mathrm{QE}$ and $\Delta$ regions are expressed in terms of

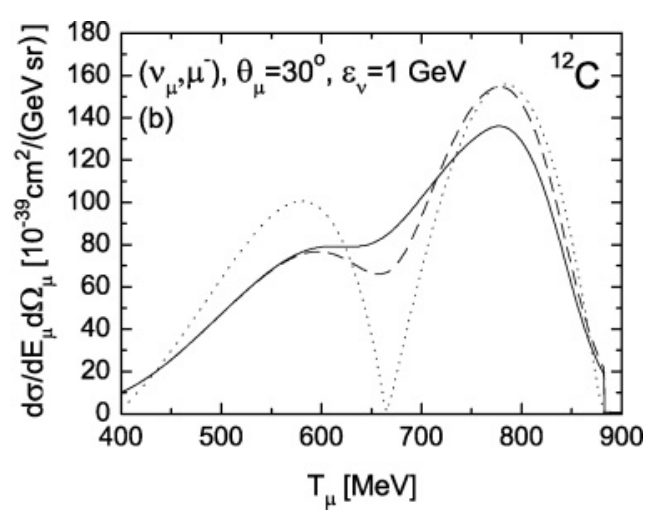

FIG. 3. The cross section of charge-changing neutrino $\left(v_{\mu}, \mu^{-}\right)$reaction on ${ }^{12} \mathrm{C}$ at $\theta_{\mu}=30^{\circ}$ and $\varepsilon_{v}=1 \mathrm{GeV}$. (a) QE contributions: the result of CDFM with $c_{1}=0.63$ (solid line); CDFM with $c_{1}=0.72$ (dashed line); RFG (dotted line); SuSA result (dot-dashed line); the result for the $\Delta$ contribution from the CDFM (double dot-dashed line). (b) the sum of QE and $\Delta$ contributions in RFG model (dotted line) and in the CDFM with $c_{1}=0.63$ (solid line) and $c_{1}=0.72$ (dashed line). Here and in the following figures the range of variation of $\psi^{\prime}$ and $\psi_{\Delta}^{\prime}$ is approximately $(-2.0,5.5)$ and $(-3.5,+2.5)$, respectively. 

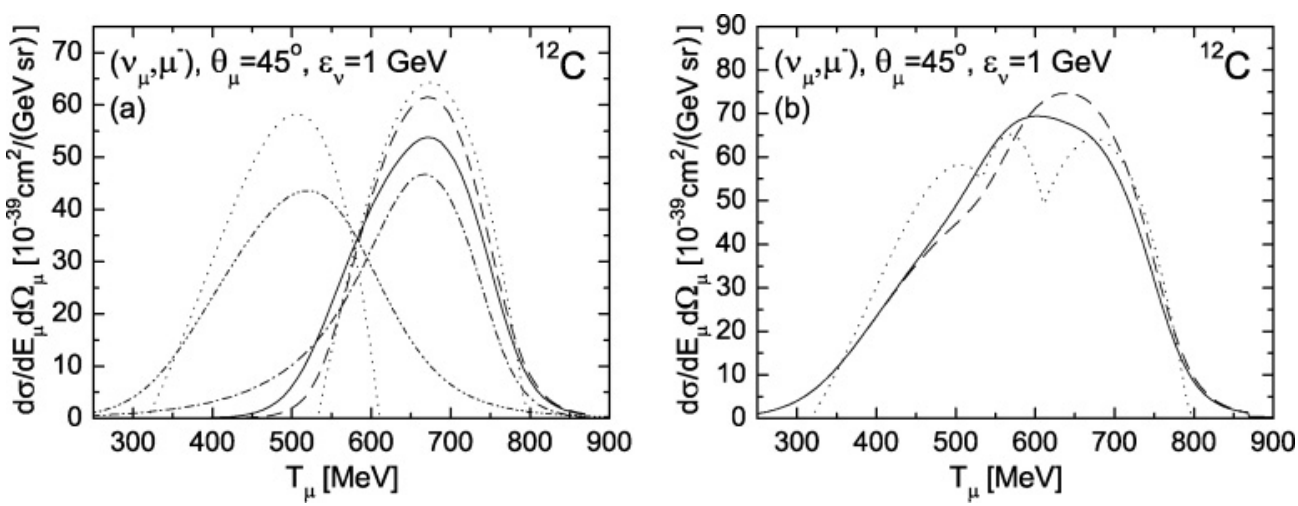

FIG. 4. The same as described in the caption to Fig. 3 for $\theta_{\mu}=45^{\circ}$ and $\varepsilon_{v}=1 \mathrm{GeV}$.
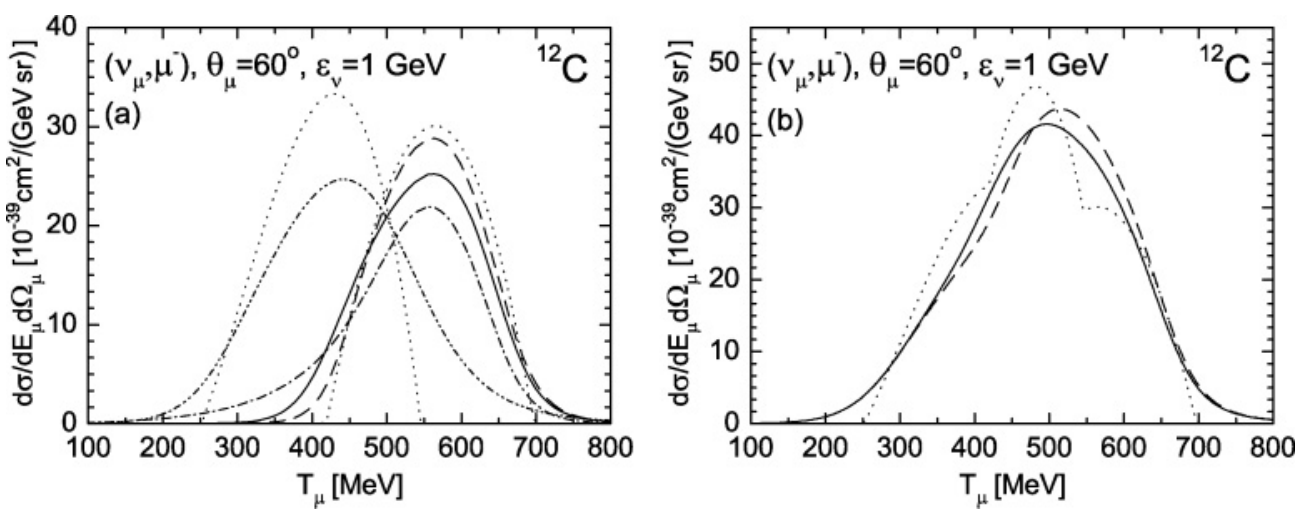

FIG. 5. The same as described in the caption to Fig. 3 for $\theta_{\mu}=60^{\circ}$ and $\varepsilon_{v}=1 \mathrm{GeV}$.
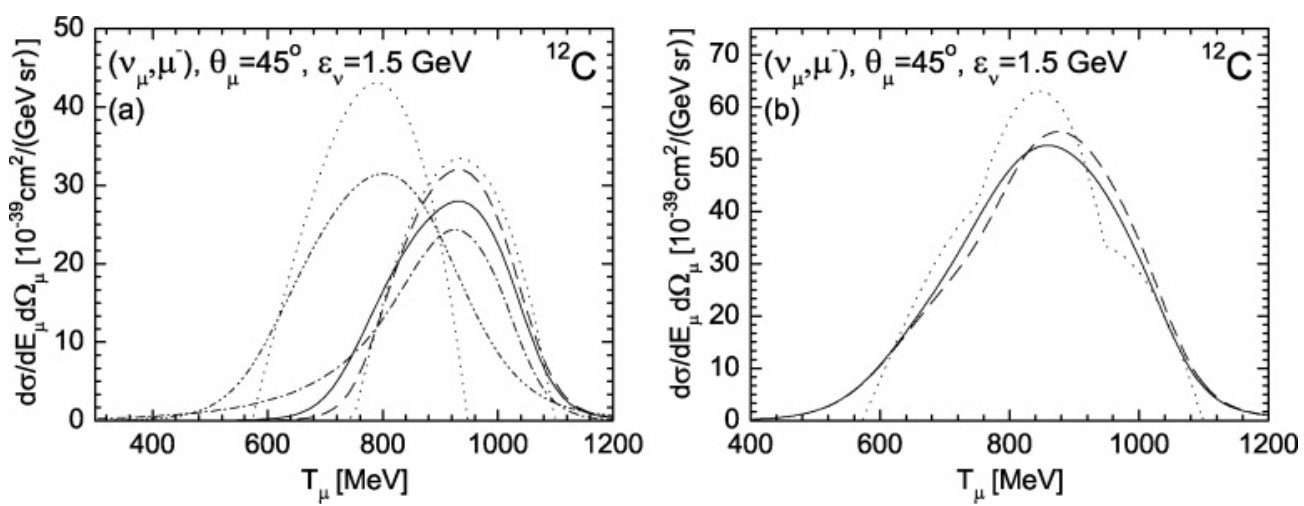

FIG. 6. The same as described in the caption to Fig. 3 for $\theta_{\mu}=45^{\circ}$ and $\varepsilon_{v}=1.5 \mathrm{GeV}$.
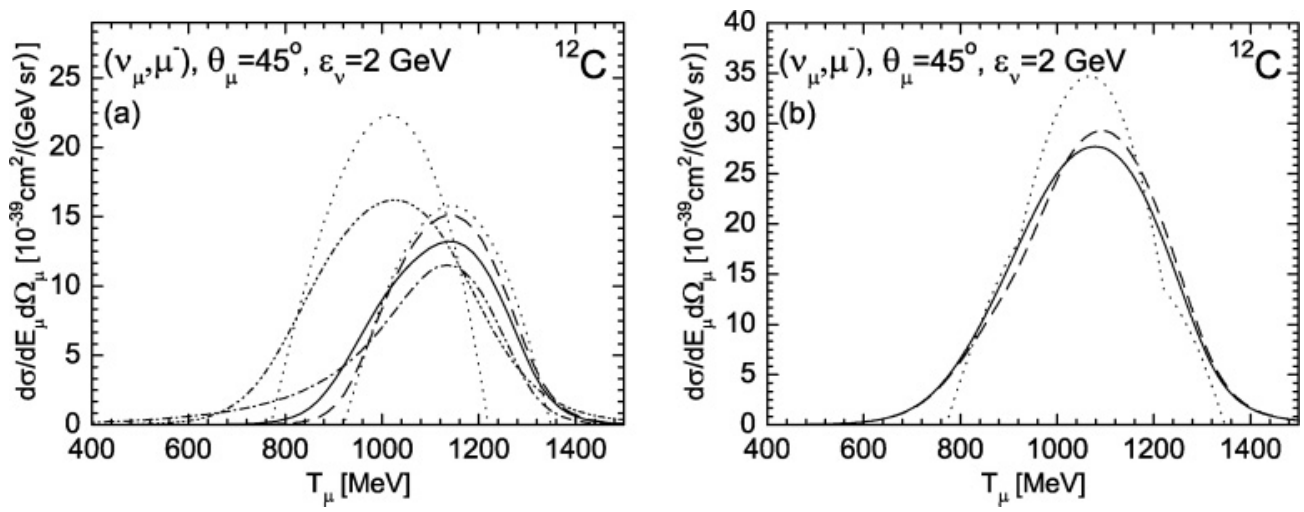

FIG. 7. The same as described in the caption to Fig. 3 for $\theta_{\mu}=45^{\circ}$ and $\varepsilon_{v}=2 \mathrm{GeV}$. 

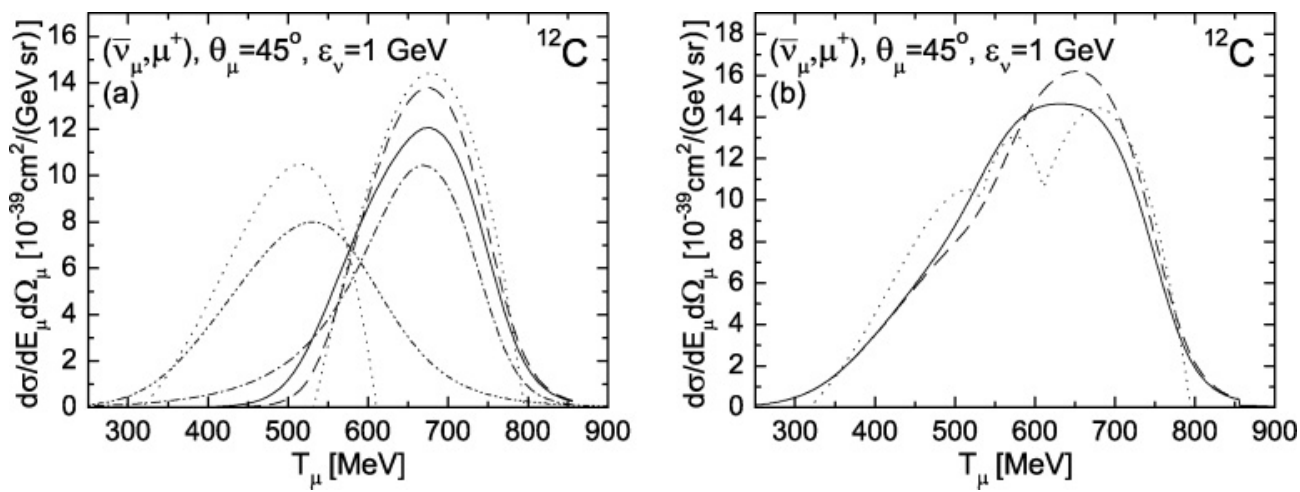

FIG. 8. The cross section of charge-changing antineutrino $\left(\bar{v}_{\mu}, \mu^{+}\right)$reaction on ${ }^{12} \mathrm{C}$ at $\theta_{\mu}=45^{\circ}$ and $\varepsilon_{v}=1 \mathrm{GeV}$. The notations are the same as described in the caption to Fig. 3.

the nuclear tensor $W^{\mu v}$ in the corresponding region, using its relationships with the RFG model scaling functions. The basic relationships used to calculate the s.n. cross sections are given in Ref. [31]. This concerns the leptonic and hadronic tensors and the response and structure functions. In our calculations of neutrino-nucleus cross sections (following Ref. [31]) we use for the nucleon form factors the Höhler parametrization 8.2 [54] in the vector sector and the form factors given in Ref. [31] in the axial-vector sector.

In the present work, instead of the RFG functions in the QE and $\Delta$ regions, we use those obtained in the CDFM (described in subsections II A and II B).

\section{RESULTS OF CALCULATIONS AND DISCUSSION}

In this section we present first the $\mathrm{QE}$ and $\triangle \mathrm{CDFM}$ scaling functions $f^{\mathrm{QE}}\left(\psi_{\mathrm{QE}}^{\prime}\right)$ and $f^{\Delta}\left(\psi_{\Delta}^{\prime}\right)$ by means of which the cross sections of CC neutrino (antineutrino) scattering on ${ }^{12} \mathrm{C}$ are calculated.

In this work we have not considered Coulomb distortion of the outgoing muon. Checks made within the effective momentum approach $[31,42,55]$ have shown that these effects are within a few percentages for the high-energy muon kinematics and the light target ${ }^{12} \mathrm{C}$ considered in this work. Therefore, our general conclusions about scaling are not modified.

In Fig. 1 we compare the $\mathrm{QE}$ and $\triangle \mathrm{CDFM}$ scaling functions, whereas in Fig. 2 a comparison of the $\Delta$-region CDFM scaling function $f^{\Delta}\left(\psi_{\Delta}^{\prime}\right)$ with the averaged experimental data for $f^{\Delta}\left(\psi_{\Delta}^{\prime}\right)$ taken from Ref. [33] (see also Fig. 5 of Ref. [19]) is given.

In Figs. 3-7 [panels 3(a)-7(a)] we give the results of calculations for cross sections (the $\mathrm{QE}$ and $\Delta$ contributions) of neutrino $\left(v_{\mu}, \mu^{-}\right)$scattering on ${ }^{12} \mathrm{C}$ at different muon angles and incident neutrino energies from 1 to $2 \mathrm{GeV}$. In the calculations we used the CDFM scaling function in the QE region \{Eqs. (1)-(6), (11)-(10), using the parabolic form [Eq. (6)] of $\left.f_{\mathrm{RFG}, 2}^{p(n)}\left[\psi^{\prime}(R)\right]\right\}$ and in the $\Delta$ region [Eqs. (15)-(28)]. The results of the CDFM in the QE case are compared with those from the RFG model and SuSA [15,31]. We present also [in panels 3(b)-7(b)] the sum of the QE and $\Delta$ contributions to the cross sections. As an example in Figs. 8(a) and 8(b) we give the results of the calculations for cross sections of antineutrino $\left(\bar{v}_{\mu}, \mu^{+}\right)$scattering on ${ }^{12} \mathrm{C}$ for the case of muon angle $\theta_{\mu}=45^{\circ}$ and the incident antineutrino energy $\varepsilon_{\bar{v}}=1 \mathrm{GeV}$.

First, it can be seen from Figs. 3-8 [panels 3(a)-8(a)] that the CDFM results (with $c_{1}=0.72$ ) for the cross sections in the QE region are close to those of the RFG model, whereas the results of CDFM (with $c_{1}=0.63$ ) are between those of RFG and SuSA. This result could be expected due to the peculiarities of the QE CDFM scaling function $f\left(\psi^{\prime}\right)$, namely that when $c_{1}=0.72$ is used it is similar to that of the RFG model (see Fig. 1), whereas when $c_{1}=0.63$ the CDFM scaling function is closer to that of the SuSA. These properties of the QE CDFM scaling function were shown in comparison with the experimental data from the electron scattering and with the RFG model and SuSA results in Fig. 6 of Ref. [53]. This consideration should be kept in mind also in relation to the observations from the CDFM analyses of the QE electron scattering (mentioned above) about the necessity to use almost symmetric scaling function $\left(c_{1}=0.72\right)$ or asymmetric one $\left(c_{1}=0.63\right)$ at different kinematical conditions. Second, we find that, in general, the strength of the QE peak decreases with increasing outgoing angle $\left(\theta_{\mu}\right)$ or with increasing incoming energy $\left(\varepsilon_{v}\right)$. Third, the height of the $\Delta$ peak also decreases with increasing $\theta_{\mu}$, but its decrease is much slower so that, at $\varepsilon_{v}=1 \mathrm{GeV}$, the relative height of the two peaks $(\Delta / \mathrm{QE})$ goes from about 0.5 at $\theta_{\mu}=30^{\circ}$ to $\geqslant 1$ at $\theta_{\mu}=60^{\circ}$. Fourth, something similar happens when we fix the angle and increase the energy. The $\Delta$ peak decreases more slowly than the QE peak. For instance, at $\theta_{\mu}=45^{\circ}$ the relative height of the two peaks goes from $\Delta / \mathrm{QE} \leqslant 1$ at $\varepsilon_{v}=1 \mathrm{GeV}$ to $\Delta / \mathrm{QE} \sim 4 / 3$ at $\varepsilon_{v}=2 \mathrm{GeV}$. Fifth, the overlap between both peaks is larger with increasing incoming energy and/or increasing scattering angle. From panels 3(b)-8(b) it can be seen for the sum of the $\mathrm{QE}$ and $\Delta$ contributions that at fixed $\theta_{\mu}$ the maximum decreases with the increase of the energy. For $\theta_{\mu}=45^{\circ}$ both CDFM curves (with $c_{1}=0.63$ and $c_{1}=0.72$ ) are quite similar for the energies $\varepsilon_{v}=1 \div 2 \mathrm{GeV}$. At energy $\varepsilon_{v}=1 \mathrm{GeV}$ and small angles (e.g., $\theta_{\mu}=30^{\circ}$ ) there are two maxima of the cross section, whereas at larger angles $\left(\theta_{\mu}=45^{\circ}\right.$ and $\left.\theta_{\mu}=60^{\circ}\right)$ the two peaks merge into one (e.g., for $\varepsilon_{v}=1 \mathrm{GeV}$ ). 
We note also that, as can be seen from Figs. 4 and 8 , similarly to the results from Ref. [31], the antineutrinoscattering cross section (for incident energy $1 \mathrm{GeV}$ and $\theta_{\mu}=45^{\circ}$ ) is about 5 times smaller than the neutrino one.

Although this is true already at the level of the RFG, the overlap region is more extended in the present model due to the tails of the corresponding scaling functions outside the RFG region $\left|\psi^{\prime}\right|<1$.

\section{CONCLUSIONS}

In our work [19] we extended the CDFM superscaling analysis [16-18] from the QE region to the $\Delta$ region of the inclusive electron scattering. In Ref. [19] the CDFM was applied also to charge-changing neutrino and antineutrino reactions at energies between 1 and $2 \mathrm{GeV}$ from ${ }^{12} \mathrm{C}$ nucleus in the quasielastic region. Later, in our work [53] we considered neutral current neutrino and antineutrino scattering with energies of $1 \mathrm{GeV}$ from ${ }^{12} \mathrm{C}$ with a proton and neutron knockout using CDFM scaling functions.

In the present work we use the $\Delta$-scaling functions obtained within the CDFM in Ref. [19] to calculate charge-changing neutrino and antineutrino scattering in the $\Delta$ region extending our previous QE analysis. So in this work we obtain both contributions (in $\mathrm{QE}$ and $\Delta$ regions) of the charge-changing neutrino scattering thus completing the CDFM analyses of both inclusive electron and neutrino scattering from nuclei on the same basis, i.e., using the same CDFM QE- and $\Delta$-region scaling functions in both cases, for incident electrons or neutrino (antineutrino). We consider the scattering of neutrino (antineutrino) with incident energies between 1 and $2 \mathrm{GeV}$ from the ${ }^{12} \mathrm{C}$ nucleus at different muon angles. Our results are compared with those from the RFG model and from SuSA [15,31]. Concerning the QE contribution to the cross section we note that the use of asymmetric CDFM scaling function $\left(c_{1}=0.63\right)$ gives results that are close to those from SuSA, whereas the symmetric scaling function $\left(c_{1}=0.72\right)$ leads to results similar with the RFG model ones.
The results for the cross sections show the following features: (i) at fixed incident energies the values of the QE- and $\Delta$-peak maxima decrease with the increase of the muon angle $\theta_{\mu}$ and the value of the $\Delta$-contribution maximum becomes closer to that of the QE contribution, (ii) at fixed angle $\theta_{\mu}$ the $\mathrm{QE}$ and $\Delta$ contributions overlap more strongly with the increase of the neutrino energy and the maximum of the $\Delta$ peak increases, and (iii) at fixed angle $\theta_{\mu}$ the maximum of the sum of both $\mathrm{QE}$ and $\Delta$ contributions to the cross section decrease with the increase of the energy. For $\theta_{\mu}=45^{\circ}$ both CDFM curves (with $c_{1}=0.63$ and $c_{1}=0.72$ ) are quite similar for the interval of neutrino energies $\varepsilon_{v}=1 \div 2 \mathrm{GeV}$. (iv) At energy $\varepsilon_{v}=1 \mathrm{GeV}$ and smaller angles (e.g., $\theta_{\mu}=30^{\circ}$ ) there are two maxima of the total sum of the $\mathrm{QE}$ and $\Delta$ contributions, whereas at larger angles $\left(\theta_{\mu}=45^{\circ}\right.$ and $\left.60^{\circ}\right)$ the two peaks merge into one (for the energy interval $\varepsilon_{v}=1 \div 2 \mathrm{GeV}$ ), and (v) similarly to the results from Ref. [31], the antineutrino cross section (on the example for incident energy $1 \mathrm{GeV}$ and muon angle of 45 degrees) is about 5 times smaller than the neutrino one.

In summary, it is pointed out that the constructed QE- and $\Delta$-region scaling functions in the CDFM can be used in a reliable way for the description of the electron and neutrino (antineutrino) scattering from nuclei.

\section{ACKNOWLEDGMENTS}

This work was partly supported by the Bulgarian National Science Fund under contract nos. $\Phi-1416$ and $\Phi-1501$ and by Ministerio de Educación y Ciencia (Spain) under contract nos. FPA2006-13807-C02-01, FIS2005-01105, and FIS200500640. This work is also partially supported by the EU program ILIAS N6 ENTApP WP1. One of the authors (M.K.G.) is grateful for the warm hospitality given by the CSIC and for support during his stay there from the State Secretariat of Education and Universities of Spain (N/Ref.SAB2005-0012).
[1] G. B. West, Phys. Rep. 18, 263 (1975).

[2] I. Sick, D. B. Day, and J. S. McCarthy, Phys. Rev. Lett. 45, 871 (1980).

[3] C. Ciofi degli Atti, E. Pace, and G. Salmè, Phys. Rev. C 36, 1208 (1987).

[4] D. B. Day, J. S. McCarthy, T. W. Donnelly, and I. Sick, Annu. Rev. Nucl. Part. Sci. 40, 357 (1990).

[5] C. Ciofi degli Atti, E. Pace, and G. Salmè, Phys. Rev. C 43, 1155 (1991).

[6] C. Ciofi degli Atti and S. Simula, Phys. Rev. C 53, 1689 (1996).

[7] C. Ciofi degli Atti and G. B. West, nucl-th/9702009.

[8] C. Ciofi degli Atti and G. B. West, Phys. Lett. B458, 447 (1999).

[9] D. Faralli, C. Ciofi degli Atti, and G. B. West, in Proceedings of 2 nd International Conference on Perspectives in Hadronic Physics, ICTP, Trieste, Italy, 1999, edited by S. Boffi, C. Ciofi degli Atti, and M. M. Giannini (World Scientific, Singapore, 2000), p. 75.

[10] W. M. Alberico, A. Molinari, T. W. Donnelly, E. L. Kronenberg, and J. W. Van Orden, Phys. Rev. C 38, 1801 (1988).
[11] M. B. Barbaro, R. Cenni, A. De Pace, T. W. Donnelly, and A. Molinari, Nucl. Phys. A643, 137 (1998).

[12] T. W. Donnelly and I. Sick, Phys. Rev. Lett. 82, 3212 (1999).

[13] T. W. Donnelly and I. Sick, Phys. Rev. C 60, 065502 (1999).

[14] C. Maieron, T. W. Donnelly, and I. Sick, Phys. Rev. C 65, 025502 (2002).

[15] M. B. Barbaro, J. A. Caballero, T. W. Donnelly, and C. Maieron, Phys. Rev. C 69, 035502 (2004).

[16] A. N. Antonov, M. K. Gaidarov, D. N. Kadrev, M. V. Ivanov, E. Moya de Guerra, and J. M. Udias, Phys. Rev. C 69, 044321 (2004).

[17] A. N. Antonov, M. K. Gaidarov, M. V. Ivanov, D. N. Kadrev, E. Moya de Guerra, P. Sarriguren, and J. M. Udias, Phys. Rev. C 71, 014317 (2005).

[18] A. N. Antonov, M. V. Ivanov, M. K. Gaidarov, E. Moya de Guerra, P. Sarriguren, and J. M. Udias, Phys. Rev. C 73, 047302 (2006). 
[19] A. N. Antonov, M. V. Ivanov, M. K. Gaidarov, E. Moya de Guerra, J. A. Caballero, M. B. Barbaro, J. M. Udias, and P. Sarriguren, Phys. Rev. C 74, 054603 (2006).

[20] A. N. Antonov, M. V. Ivanov, M. K. Gaidarov, and E. Moya de Guerra, Phys. Rev. C 75, 034319 (2007).

[21] O. Benhar, D. Day, and I. Sick, Rev. Mod. Phys. 80, 189 (2008).

[22] L. Alvarez-Ruso, M. B. Barbaro, T. W. Donnelly, and A. Molinari, Nucl. Phys. A724, 157 (2003).

[23] J. E. Amaro, M. B. Barbaro, J. A. Caballero, T. W. Donnelly, and A. Molinari, Nucl. Phys. A697, 388 (2002); A723, 181 (2003); Phys. Rep. 368, 317 (2002).

[24] A. De Pace, M. Nardi, W. M. Alberico, T. W. Donnelly, and A. Molinari, Nucl. Phys. A726, 303 (2003); A741, 249 (2004).

[25] J. E. Amaro, M. B. Barbaro, J. A. Caballero, T. W. Donnelly, and A. Molinari, Nucl. Phys. A643, 349 (1998).

[26] J. E. Amaro, M. B. Barbaro, J. A. Caballero, T. W. Donnelly, and A. Molinari, Nucl. Phys. A723, 181 (2003).

[27] J. E. Amaro, M. B. Barbaro, J. A. Caballero, and T. W. Donnelly, Phys. Rev. C 73, 035503 (2006).

[28] A. N. Antonov, V. A. Nikolaev, and I. Zh. Petkov, Bulg. J. Phys. 6, 151 (1979); Z. Phys. A 297, 257 (1980); 304, 239 (1982); Nuovo Cimento A 86, 23 (1985); 102, 1701 (1989); A. N. Antonov, D. N. Kadrev, and P. E. Hodgson, Phys. Rev. C 50, 164 (1994).

[29] A. N. Antonov, P. E. Hodgson, and I. Zh. Petkov, Nucleon Momentum and Density Distributions in Nuclei (Clarendon Press, Oxford, 1988); Nucleon Correlations in Nuclei (SpringerVerlag, Berlin, 1993).

[30] J. J. Griffin and J. A. Wheeler, Phys. Rev. 108, 311 (1957).

[31] J. E. Amaro, M. B. Barbaro, J. A. Caballero, T. W. Donnelly, A. Molinari, and I. Sick, Phys. Rev. C 71, 015501 (2005); M. B. Barbaro, Nucl. Phys. B, Proc. Suppl. 159, 186 (2006); M. B. Barbaro, J. E. Amaro, J. A. Caballero, and T. W. Donnelly, in Nuclear Theory: Proceedings of 25th International Workshop on Nuclear Theory, Rila Mountains, Bulgaria, June 26-July 1, 2006, edited by S. Dimitrova (DioMira, Sofia, 2006), p. 73.

[32] J. Jourdan, Nucl. Phys. A603, 117 (1996).

[33] J. A. Caballero, J. E. Amaro, M. B. Barbaro, T. W. Donnelly, C. Maieron, and J. M. Udias, Phys. Rev. Lett. 95, 252502 (2005).

[34] J. A. Caballero, Phys. Rev. C 74, 015502 (2006).

[35] J. E. Amaro, M. B. Barbaro, J. A. Caballero, T. W. Donnelly, and J. M. Udias, Phys. Rev. C 75, 034613 (2007).

[36] Y. Fukuda et al. (The Super-Kamiokande Collaboration), Phys. Rev. Lett. 81, 1562 (1998); M. H. Ahn et al. (K2K Collaboration), ibid. 90, 041801 (2003); Q.-R. Ahmad et al. (SNO Collaboration), ibid. 87, 071301 (2001); 89, 011301 (2002); K. Eguchi et al. (KamLAND Collaboration), ibid. 90, 021802 (2003); C. Athanassopoulos et al. (LSND Collaboration), ibid. 77, 3082 (1996); 81, 1774 (1998).
[37] J. E. Amaro, M. B. Barbaro, J. A. Caballero, T. W. Donnelly, and C. Maieron, Phys. Rev. C 71, 065501 (2005).

[38] J. A. Caballero, J. E. Amaro, M. B. Barbaro, T. W. Donnelly, and J. M. Udias, Phys. Lett. B653, 366 (2007).

[39] M. C. Martinez, P. Lava, N. Jachowicz, J. Ryckebusch, K. Vantournhout, and J. M. Udias, Phys. Rev. C 73, 024607 (2006).

[40] J. Nieves, M. Valverde, and M. J. Vicente-Vacas, Nucl. Phys. B, Proc. Suppl. 155, 263 (2006); nucl-th/0510010; J. Nieves, M. Valverde, and M. J. Vicente Vacas, Phys. Rev. C 73, 025504 (2006).

[41] M. B. Barbaro, J. E. Amaro, J. A. Caballero, T. W. Donnelly, and A. Molinari, Nucl. Phys. B, Proc. Suppl. 155, 257 (2006).

[42] C. Maieron, M. C. Martinez, J. A. Caballero, and J. M. Udias, Phys. Rev. C 68, 048501 (2003).

[43] A. Meucci, C. Giusti, and F. D. Pacati, Nucl. Phys. A739, 277 (2004); A773, 250 (2006).

[44] O. Benhar, Nucl. Phys. B, Proc. Suppl. 139, 15 (2005); O. Benhar and N. Farina, Nucl. Phys. B, Proc. Suppl. 139, 230 (2005); O. Benhar, N. Farina, H. Nakamura, M. Sakuda, and R. Seki, Nucl. Phys. B, Proc. Suppl. 155, 254 (2006); O. Benhar, N. Farina, H. Nakamura, M. Sakuda, and R. Seki, Phys. Rev. D 72, 053005 (2005).

[45] G. Co', Nucl. Phys. B, Proc. Suppl. 159, 192 (2006); A. Botrugno and G. Co', Nucl. Phys. A761, 200 (2005); M. Martini, G. Co', M. Anguiano, and A. M. Lallena, Phys. Rev. C 75, 034604 (2007).

[46] T. Leitner, L. Alvarez-Ruso, and U. Mosel, Phys. Rev. C 73, 065502 (2006).

[47] B. Szczerbinska, T. Sato, K. Kubodera, and T.-S. H. Lee, Phys. Lett. B649, 132 (2007).

[48] O. Buss, T. Leitner, U. Mosel, and L. Alvarez-Ruso, Phys. Rev. C 76, 035502 (2007).

[49] A. Meucci, C. Giusti, and F. D. Pacati, Nucl. Phys. A744, 307 (2004).

[50] W. M. Alberico and C. Maieron, arXiv: hep-ph/0210017 (2002).

[51] T. Leitner, L. Alvarez-Ruso, and U. Mosel, Phys. Rev. C 74, 065502 (2006).

[52] K. S. Kim, B. G. Yu, M. K. Cheoun, T. K. Choi, and M. T. Chung, arXiv:0707.1370 [nucl-th] (2007).

[53] A. N. Antonov, M. V. Ivanov, M. B. Barbaro, J. A. Caballero, E. Moya de Guerra, and M. K. Gaidarov, Phys. Rev. C 75, 064617 (2007).

[54] G. Höhler, E. Pietarinen, I. Sabba-Stefanescu, F. Borkowski, G. G. Simon, V. H. Walther, and R. D. Wendling, Nucl. Phys. B114, 505 (1976).

[55] W. M. Alberico, M. B. Barbaro, S. M. Bilenky, J. A. Caballero, C. Giunti, C. Maieron, E. Moya de Guerra, and J. M. Udias, Nucl. Phys. A 623, 471 (1997). 